\title{
Proton fire hose instabilities in the expanding solar wind
}

\author{
Petr Hellinger ${ }^{1,2, \dagger}$ \\ ${ }^{1}$ Astronomical Institute, CAS, Bocni II/1401, CZ-14000 Prague, Czech Republic \\ ${ }^{2}$ Institute of Atmospheric Physics, CAS, Bocni II/1401, CZ-14000 Prague, Czech Republic
}

(Received 7 November 2016; revised 23 January 2017; accepted 24 January 2017)

Using two-dimensional hybrid expanding box simulations we study the competition between the continuously driven parallel proton temperature anisotropy and fire hose instabilities in collisionless homogeneous plasmas. For a quasi-radial ambient magnetic field the expansion drives $T_{p \|}>T_{p \perp}$ and the system becomes eventually unstable with respect to the dominant parallel fire hose instability. This instability is generally unable to counteract the induced anisotropization and the system typically becomes unstable with respect to the oblique fire hose instability later on. The oblique instability efficiently reduces the anisotropy and the system rapidly stabilizes, while a significant part of the generated electromagnetic fluctuations is damped to protons. As long as the magnetic field is in the quasi-radial direction, this evolution repeats itself and the electromagnetic fluctuations accumulate. For a sufficiently oblique magnetic field the expansion drives $T_{p \perp}>T_{p \|}$ and brings the system to the stable region with respect to the fire hose instabilities.

Key words: astrophysical plasmas, plasma expansion, plasma simulation

\section{Introduction}

In situ observations in the weakly collisional solar wind (Marsch 2006) indicate the presence of apparent bounds on different particle parameters. The observed bounds on the temperature anisotropy of protons (Gary et al. 2001; Kasper et al. 2003; Hellinger et al. 2006; Hellinger \& Trávníček 2014), alpha particles (Maruca, Kasper \& Gary 2012), electrons (Stverák et al. 2008), and/or on the differential velocity between ion species (Bourouaine et al. 2013; Matteini et al. 2015) and the differential velocity between different electron species (heat flux driven instabilities) (Gary et al. 1999) are often compatible with the constraints imposed by kinetic plasma instabilities. Some observations also indicate that there are enhanced levels of fluctuations near the apparent bounds (Bale et al. 2009; Wicks et al. 2013; Lacombe et al. 2014), supporting the interpretation that these bounds are imposed by the kinetic instabilities.

The evolution of the solar wind particle properties depends on the processes that are active. For instance, Alfvén ion cyclotron waves heat the ions in the direction perpendicular to the ambient magnetic field (Hollweg \& Isenberg 2002;

$\dagger$ Email address for correspondence: Petr.Hellinger@asu.cas.cz 
Hellinger et al. 2005), whereas plasma turbulence generates a range of particle temperature anisotropies and even non-gyrotropies (Servidio et al. 2015). The solar wind expansion tends to generate particle temperature anisotropies and influences as well the differential streaming between different species. Matteini et al. (2007) investigated the radial evolution of the proton temperature anisotropy in the fast solar wind in the beta-anisotropy plane: below 1 a.u., protons follow an anticorrelation between the proton beta and the proton temperature anisotropy (cf. Marsch, Ao \& Tu 2004). This behaviour is not compatible with the double-adiabatic prediction (Chew, Goldberger \& Low 1956) and indicates the presence of proton heating, likely owing to the turbulent cascade (Cranmer et al. 2009; Hellinger et al. 2013). Beyond 1 a.u., the results of Matteini et al. (2007) indicate that the behaviour of protons changes; the system seems to follow in the beta-anisotropy plane a marginal stability path with respect to the proton fire hose instabilities.

Results similar to the observations of Matteini et al. (2007) are seen in numerical simulations using the expanding box model (Matteini et al. 2006; Hellinger \& Trávníček 2008): the expansion drives the parallel temperature anisotropy and fire hose instabilities limit the accessible temperature anisotropy. These results were, however, obtained for the strictly radial ambient magnetic field, whereas the magnetic field in the solar wind is generally oblique with respect to the radial direction and rotates towards a transverse direction (Parker 1958). For a solar wind magnetic field which is sufficiently oblique relative to the radial direction from the Sun, the double-adiabatic approximation predicts generation of the perpendicular temperature anisotropy (Matteini et al. 2012). In this paper we investigate the role of the oblique magnetic field on the fire hose instabilities using two-dimensional hybrid expanding box simulations. The paper is organized as follows: $\S 2$ presents the simulation model $(\S 2.1)$ and the results for the radial magnetic field for different expansion times $(\S \S 2.2$ and 2.3). Section 2.4 describes the results for the Parker spiral magnetic field. Finally, §3 summarizes and discusses the simulation results.

\section{Simulation results}

\subsection{Expanding box model}

Here we use the expanding box model (Grappin, Velli \& Mangeney 1993; Grappin \& Velli 1996) implemented to the hybrid code developed by Matthews (1994) to study the response of a collisionless plasma to a slow expansion. In this hybrid expanding box (HEB) model the expansion is described as an external force; one assumes a solar wind with a constant radial velocity $v_{s w}$. Transverse scales (with respect to the radial direction) of a small portion of plasma, co-moving with the solar wind velocity, increase with time as $1+t / t_{e}$, where $t_{e}=R_{0} / v_{s w}$ is the (initial) characteristic expansion time $\left(R_{0}\right.$ being the initial radial distance). The expanding box uses these co-moving coordinates, the physical transverse scales of the simulation box increase with time (see Hellinger \& Trávníček 2005, for a detailed description of the code), and standard periodic boundary conditions are used.

The characteristic spatial and temporal units used in the model are the initial proton inertial length $d_{p 0}=c / \omega_{p p 0}$ and the inverse initial proton cyclotron frequency $1 / \omega_{c p 0}$. Here $c$ is the speed of light, $\omega_{p p 0}=\left(n_{p 0} e^{2} / m_{p} \epsilon_{0}\right)^{1 / 2}$ is the initial proton plasma frequency, $\omega_{c p 0}=e B_{0} / m_{p}, B_{0}$ is the initial magnitude of the ambient magnetic field $\boldsymbol{B}_{0}, n_{p 0}$ is the initial proton density, $e$ and $m_{p}$ are the proton electric charge and mass, respectively; finally, $\epsilon_{0}$ is the dielectric permittivity of vacuum. We use a spatial resolution $\Delta x=\Delta y=0.5 d_{p 0}$, and there are initially 4096 particles per cell for protons. 
Fields and moments are defined on a $2048 \times 10242 \mathrm{D}$ grid (unless stated otherwise) Protons are advanced using the Boris scheme with a time step $\Delta t=0.05 / \omega_{c p 0}$, while the magnetic field $\boldsymbol{B}$ is advanced with a smaller time step $\Delta t_{B}=\Delta t / 10$. The initial ambient magnetic field is in the $2 \mathrm{D}$ simulation box, $\boldsymbol{B}_{0}=B_{0}\left(\cos \theta_{B R}, \sin \theta_{B R}, 0\right)$ with the initial angle between the magnetic field and the radial direction $\theta_{B R}=0^{\circ}$ and $32^{\circ}$. Initially we set $\beta_{p \|}=2.4$ and $T_{p \perp} / T_{p \|}=0.7$ to start the simulation close to the unstable region and we set the expansion time $t_{e}=10^{4} / \omega_{c p 0}$ (unless stated otherwise).

\subsection{Radial magnetic field}

We start with the well-studied case with the strictly radial magnetic field (Matteini et al. 2006; Hellinger \& Trávníček 2008). In this case the expansion continuously drives $T_{p \|}>T_{p \perp}$. When no wave activity/turbulence is present, the system is collisionless, and no important heat flux exists one expects that the plasma system follows the double-adiabatic/CGL prediction (Chew et al. 1956) for the parallel and perpendicular proton temperatures

$$
\left.T_{p \|}\right|_{C G L} \propto \frac{n^{2}}{B^{2}} \quad \text { and }\left.\quad T_{p \perp}\right|_{C G L} \propto B,
$$

respectively. For the radial magnetic field we have $B \propto R^{-2}$ and $n \propto R^{-2}$, so that $T_{p \|}$ is constant and $T_{p \perp} \propto R^{-2}$.

Figure 1 displays the evolution in the 2D HEB simulation for the radial magnetic field $\left(\theta_{B R}=0^{\circ}\right)$ : the solid curve shows the path in the space $\left(\beta_{p \|}, T_{p \perp} / T_{p \|}\right)$; the empty circle denotes the initial condition. The dashed contours show the linear prediction in a homogeneous plasma with bi-Maxwellian protons with respect to the (blue) parallel and (red) oblique fire hoses; the dotted curve displays the corresponding double-adiabatic/CGL prediction. The system initially follows the CGL prediction $\beta_{p \|} \propto R^{2}, T_{p \perp} / T_{p \|} \propto R^{-2}$. When the system enters the region unstable with respect to the parallel fire hose (Gary et al. 1998), the generated waves scatter protons and reduce the parallel temperature, which leads to relatively weak departures from the CGL prediction. The system eventually enters the region unstable with respect to the oblique fire hose (Hellinger \& Matsumoto 2000). The plasma then jumps towards the stable region and after that a CGL-like behaviour reappears and the parallel temperature anisotropy increases. This is stopped by another jump that strongly reduces the theoretical instability growth rates. The oscillations between weakly and strongly unstable regions repeat till the end of the simulations.

The oscillatory behaviour is connected with the self-destructive properties of the oblique fire hose. Figure 2 shows the evolution of the fluctuating magnetic energy. Figure 2(a) shows the fluctuating magnetic energy $\delta B^{2} / B_{0}^{2}$ as a function of time. Figure $2(b, c)$ show colour scale plots of the fluctuating magnetic energy $\delta B^{2}$ as a function of time and the wave vector $\boldsymbol{k}$ and function of time and angle $\theta_{k B}$ between the ambient magnetic field and the wave vector, respectively. As the system expands the plasma becomes unstable with respect to the parallel fire hose and relatively weak $\left(\delta B^{2} / B_{0}^{2} \sim 0.0004\right)$ quasi-parallel $\left(\theta_{k B} \sim 0^{\circ}\right)$ propagating modes appear at proton scales $\left(k \sim 0.5 d_{p}^{-1}\right)$ for $0.10 \lesssim t / t_{e} \lesssim 0.15$. Around $t \sim 0.4$ oblique fire hose wave activity appears and mostly disappears quite rapidly (on a time scale $\sim 0.1 t_{e}$ ). This self-destructive behaviour is connected with the dispersive properties of the oblique fire hose instability: it destabilizes non-propagating oblique modes that only exist for sufficiently anisotropic protons and, as the generated waves scatter protons and reduce 


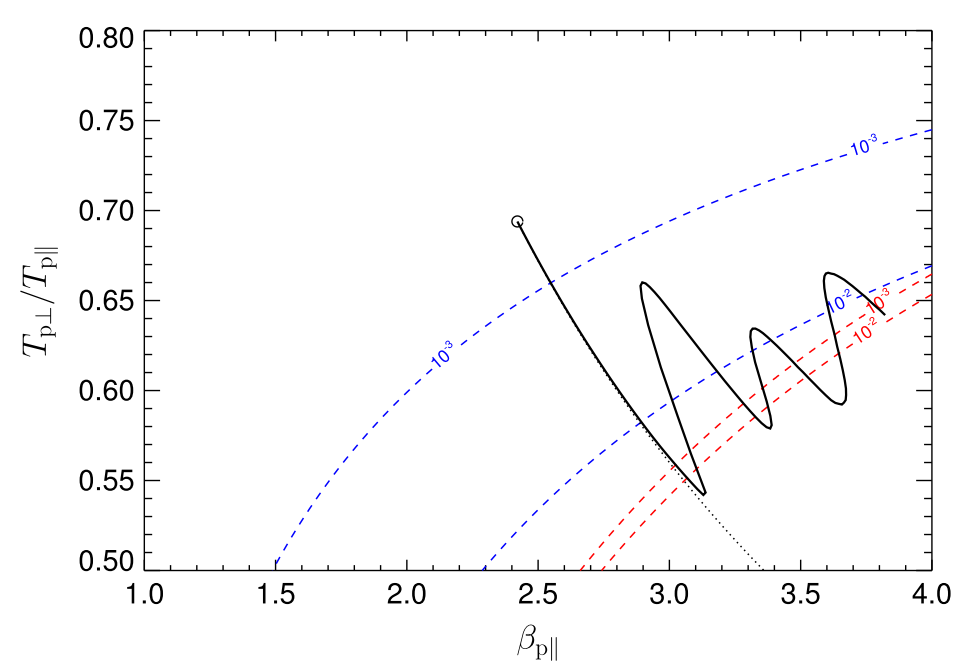

FIgURE 1. Evolution in 2D HEB simulation for the radial $\boldsymbol{B}$ and $t_{e} \omega_{c p 0}=10^{4}$ : the path in the space $\left(\beta_{p \|}, T_{p \perp} / T_{p \|}\right)$ is shown by the solid curve; the empty circle denotes the initial condition. The dashed contours show the linear prediction in a homogeneous plasma with bi-Maxwellian protons, the maximum growth rate (in units of $\omega_{c p}$ ) as a function of $\beta_{p \|}$ and $T_{p \perp} / T_{p \|}$ for (blue) the parallel proton fire hose and (red) the oblique one. The dotted curve displays the corresponding double-adiabatic prediction.

the temperature anisotropy, the non-propagating modes disappear and the fluctuations transform to damped oblique Alfvén waves (Hellinger \& Matsumoto 2000, 2001). The system with a relatively weak level of wave activity then follows a CGL-like evolution leading to the generation of the parallel temperature anisotropy and again (mostly oblique) fire hose activity appears leading to the quasi-periodic oscillations in $\delta B$ and in the space $\left(\beta_{p \|}, T_{p \perp} / T_{p \|}\right)$.

For the radial magnetic field we recover the previous results (Matteini et al. 2006; Hellinger \& Trávníček 2008). The expansion time we used in the HEB simulations is at least 10 times faster than what is typically observed in the solar wind. It is important to investigate the role of the expansion time.

\subsection{Role of the expansion time}

We performed another simulation with $t_{e} \omega_{c p 0}=10^{5}$. This value is close to realistic expansion times in the vicinity of 1 a.u. In order to be able to perform a simulation on such much longer time scales compared to the previous case, we reduced the size of the simulation box to $512 \times 512$ (while keeping the same resolution and the same initial plasma parameters). We also increased the number of particles per cell to 16384 to reduce the noise level, so that the corresponding numerical particle scattering is weak on the expansion time scale.

One expects that for faster expansion the system enters further into the unstable regions and generates stronger wave activity to counteract the expansion-driven anisotropization (Hellinger \& Trávníček 2005; Matteini et al. 2006). Figure 3 shows the evolution in the $2 \mathrm{D} \mathrm{HEB}$ simulation for the radial magnetic field $\left(\theta_{B R}=0^{\circ}\right)$ and $t_{e} \omega_{c p 0}=10^{5}$ in the same format as in figure 1: the solid curve displays the path in the space $\left(\beta_{p \|}, T_{p \perp} / T_{p \|}\right)$; the empty circle denotes the initial condition. 


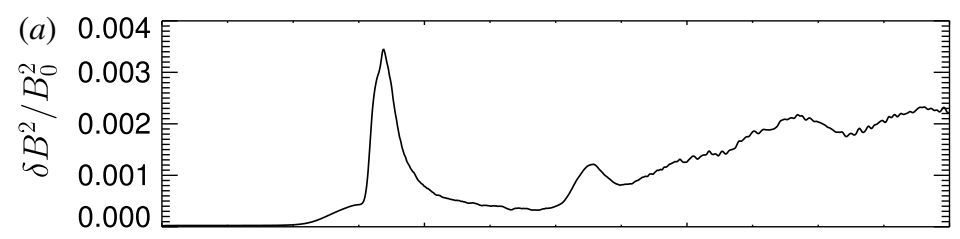

(b)
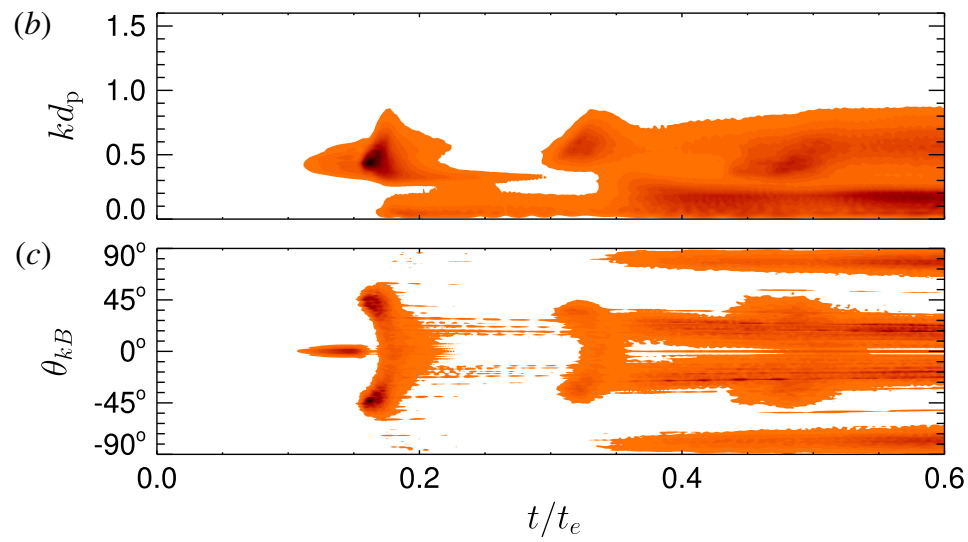

FIGURE 2. Evolution in 2D HEB simulation for the radial $\boldsymbol{B}$ and $t_{e} \omega_{c p 0}=10^{4}$. (a) Fluctuating magnetic energy $\delta B^{2} / B_{0}^{2}$ as a function of time. (b) Colour scale plot of the fluctuating magnetic energy $\delta B^{2}$ as a function of time and the wave vector $\boldsymbol{k}$. (c) Colour scale plot of the fluctuating magnetic energy $\delta B^{2}$ as a function of time and angle $\theta_{k B}$.

The dashed contours show the linear prediction in a homogeneous plasma with bi-Maxwellian protons for (blue) the parallel and (red) the oblique fire hoses. The dotted curve displays the corresponding double-adiabatic/CGL prediction. Figure 3 indeed demonstrates that the system with the slower expansion enters less far into the unstable region. Moreover, this plot suggests that the system does not enter inside the region unstable with respect to the oblique fire hose. On the other hand, the observed oscillations suggest that the oblique fire hose was active.

Figure 4 shows the evolution of the fluctuating magnetic energy in the same format as in figure 2. Figure $4(a)$ shows the fluctuating magnetic energy $\delta B^{2} / B_{0}^{2}$ as a function of time. Figure $4(b, c)$ show colour scale plots of the fluctuating magnetic energy $\delta B^{2}$ as a function of time and the wave vector $\boldsymbol{k} d_{p}$ and as a function of time and angle $\theta_{k B}$ between the ambient magnetic field and the wave vector, respectively.

The evolution is similar to that seen in the simulation with the 10-times faster expansion (see $\S 2.2$ ). The expansion drives continuously $T_{p \|}>T_{p \perp}$ following the CGL prediction. When the system enters the region unstable to the parallel fire hose, the generated wave activity affects the evolution causing small departures from the CGL prediction. When oblique fire hose activity appears, the system rapidly moves to the less unstable region while a large portion of generated wave activity is damped. After that, the system again evolves in a CGL-like manner and the process repeats quasiperiodically.

The comparison between the two simulations demonstrates that the evolutions for $t_{e} \omega_{c p 0}=10^{4}$ and $t_{e} \omega_{c p 0}=10^{5}$ are similar (qualitatively and semiquantitatively). The main difference is that the amplitude of fluctuations is smaller for the slower expansion and that the system enters further into the unstable region for the faster expansion. In the case of $t_{e} \omega_{c p 0}=10^{5}$, the bi-Maxwellian linear theory predicts that 


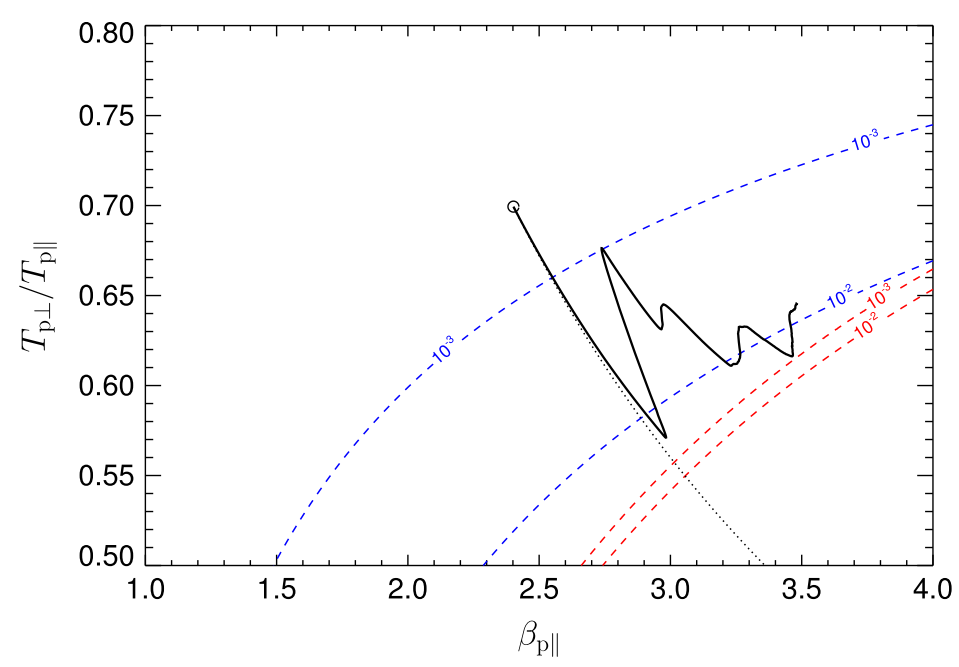

FIGURE 3. Evolution in 2D HEB simulation for the radial $\boldsymbol{B}$ and $t_{e} \omega_{c p 0}=10^{5}$ : the path in the space $\left(\beta_{p \|}, T_{p \perp} / T_{p \|}\right)$ is shown by the solid curve; the empty circle denotes the initial condition. The dashed contours show the linear prediction in a homogeneous plasma with bi-Maxwellian protons, the maximum growth rate (in units of $\omega_{c p}$ ) as a function of $\beta_{p \|}$ and $T_{p \perp} / T_{p \|}$ for (blue) the parallel proton fire hose and (red) the oblique one. The dotted curve displays the corresponding double-adiabatic prediction.

the system remains stable with respect to the oblique fire hose but in the simulation an important oblique fire hose activity appears. We will look at this phenomenon in detail in the next subsection, in the case of the oblique magnetic field.

\subsection{Oblique magnetic field}

The magnetic field in the solar wind is usually oblique with respect to the radial direction. For the expanding box approximation we have from the conservation of the magnetic flux for radial and transverse components of the magnetic field

$$
B_{r} \propto R^{-2} \quad \text { and } \quad B_{t} \propto R^{-1},
$$

respectively, in agreement with the ideal Parker spiral model (Parker 1958). For a magnetic field close to the radial direction the expansion drives the parallel anisotropy, $T_{p \|}>T_{p \perp}$, but for a sufficiently oblique magnetic field $\left(\theta_{B R} \gtrsim 45^{\circ}\right)$ the opposite anisotropy is generated. In this subsection, we investigate this transition starting with $\theta_{B R}=32^{\circ}$. This particular value of $\theta_{B R}$ corresponds to the radial distance $R \sim 0.6$ a.u. and was chosen in order to have initially a sufficiently long time of the generation of $T_{p \|}>T_{p \perp}$ and a relatively long phase where $T_{p \|}<T_{p \perp}$ is generated at later times of the simulations. The duration of this simulation is $t_{\max }=2 t_{e}$, so that at the end $R \sim 1.8$ a.u. and the ambient magnetic field angle with the radial direction is $\theta_{B R} \sim 62^{\circ}$.

Figure 5 shows the evolution in the 2D HEB simulation with $\theta_{B R}=32^{\circ}$ in the same format as in figures 1 and 3: the solid curve displays the path in the space $\left(\beta_{p \|}, T_{p \perp} / T_{p \|}\right)$; the empty circle denotes the initial condition. The dashed contours show the linear prediction in a homogeneous plasma with bi-Maxwellian protons, the maximum growth rate (in units of $\omega_{c p}$ ) as a function of $\beta_{p \|}$ and $T_{p \perp} / T_{p \|}$ for (blue) the parallel proton fire hose and (red) the oblique one. The dotted curve displays 


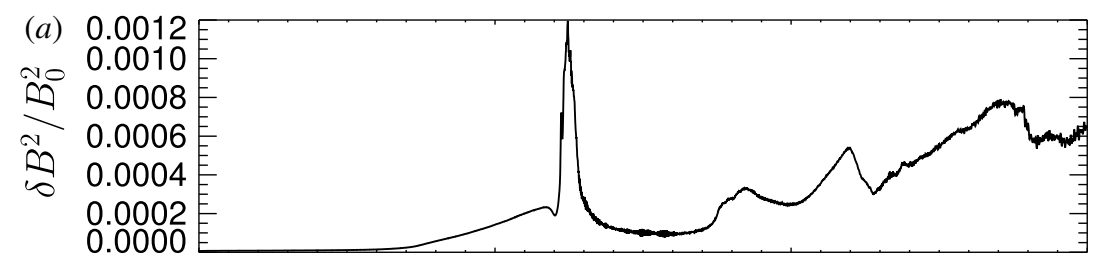

(b)

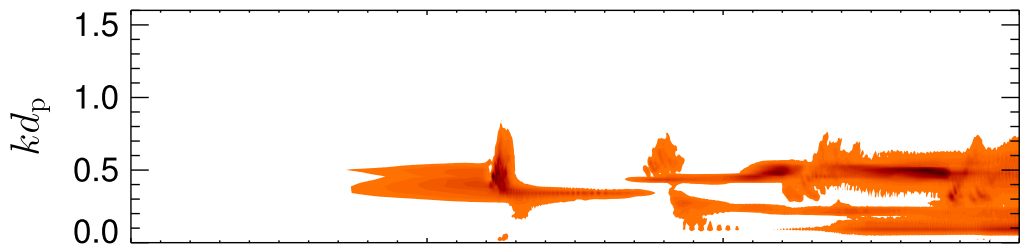

(c)

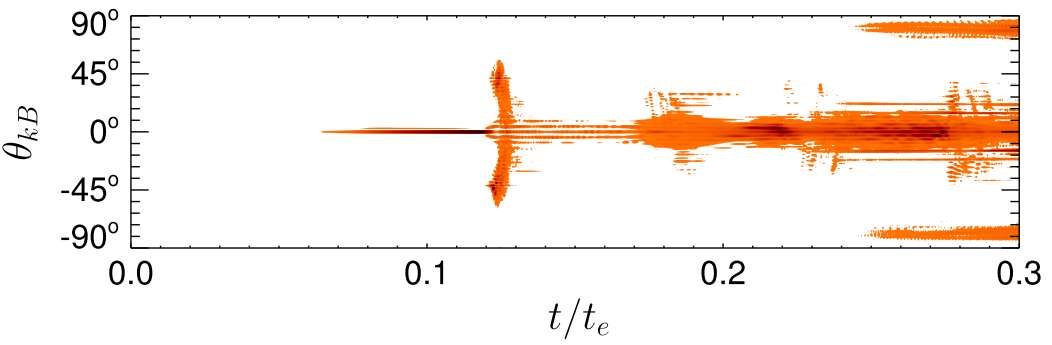

FIGURE 4. Evolution in 2D HEB simulation with the radial magnetic field and $t_{e} \omega_{c p 0}=$ $10^{5}$. (a) Fluctuating magnetic energy $\delta B^{2} / B_{0}^{2}$ as a function of time. (b) Colour scale plot of the fluctuating magnetic energy $\delta B^{2}$ as a function of time and the wave vector $\boldsymbol{k}$. (c) Colour scale plot of the fluctuating magnetic energy $\delta B^{2}$ as a function of time and angle $\theta_{k B}$.

the double-adiabatic prediction. The system initially follows the double-adiabatic prediction but when it enters the region unstable with respect to the parallel fire hose this behaviour is slightly affected. The presence of this instability is not sufficient to overcome the temperature anisotropization owing to the expansion in this case. The system enters less far into the unstable region compared to the radial case with the same expansion time (see figure 1) as the anisotropization in the case of the oblique magnetic field is weaker. The plasma does not enter into the region unstable with respect to the oblique fire hose in the corresponding bi-Maxwellian plasma. However, the path in $\left(\beta_{p \|}, T_{p \perp} / T_{p \|}\right)$ exhibits a return movement along almost the same CGL trajectory to the less unstable region, likely due to the destabilization of the oblique fire hose as in the previous subsection. After this, the system follows a double-adiabatic-like path, just shifted in the $\left(\beta_{p \|}, T_{p \perp} / T_{p \|}\right)$ space.

A more detailed comparison between the simulation results and the double-adiabatic prediction is shown in figure 6 that displays the proton parallel $T_{p \|}(a)$ and perpendicular $T_{p \perp}(b)$ as functions of time compared to the prediction. Until about $t=0.4 t_{e}$, both the temperatures follow quite closely the double-adiabatic prediction, while around this time there is an important reduction of the parallel temperature and enhancement of the perpendicular one compared to the double-adiabatic prediction. After that, the system follows the double-adiabatic prediction again.

The disruption of the double-adiabatic prediction is caused by the generation of the oblique fire hose fluctuations. Figure 7 shows the evolution of the fluctuating magnetic energy. Figure $7(a)$ shows the fluctuating magnetic energy $\delta B^{2} / B_{0}^{2}$ as a function of 


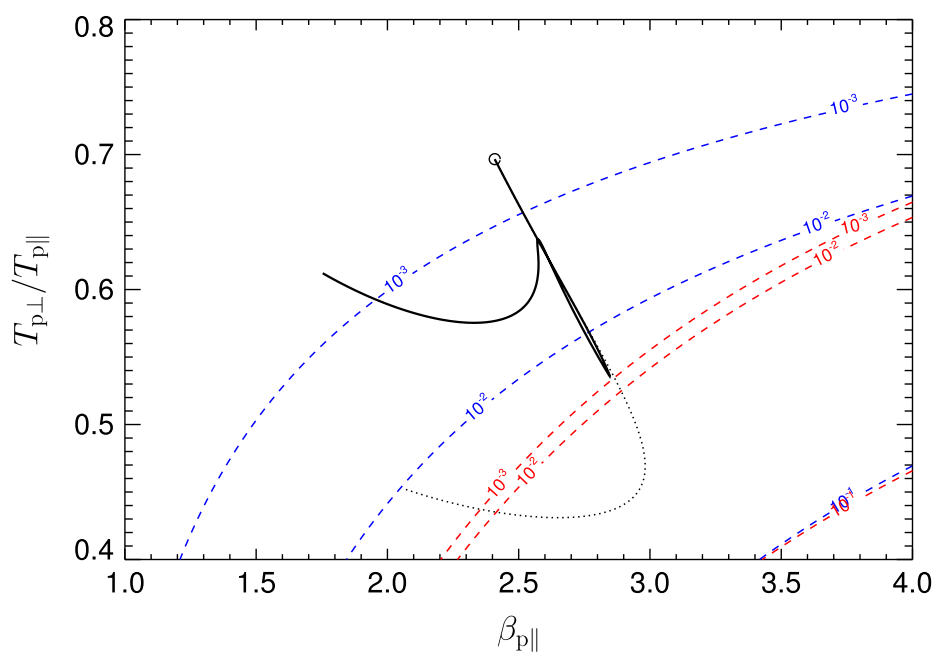

FIgURE 5. Evolution in 2D HEB simulation for $\theta_{B R}=32^{\circ}$ and $t_{e} \omega_{c p 0}=10^{4}$ : the path in the space $\left(\beta_{p \|}, T_{p \perp} / T_{p \|}\right)$ is shown by the solid curve; the empty circle denotes the initial condition. The dashed contours show the linear prediction in a homogeneous plasma with bi-Maxwellian protons, the maximum growth rate (in units of $\omega_{c p}$ ) as a function of $\beta_{p \|}$ and $T_{p \perp} / T_{p \|}$ for (blue) the parallel proton fire hose and (red) the oblique one. The dotted curve displays the corresponding double-adiabatic prediction.

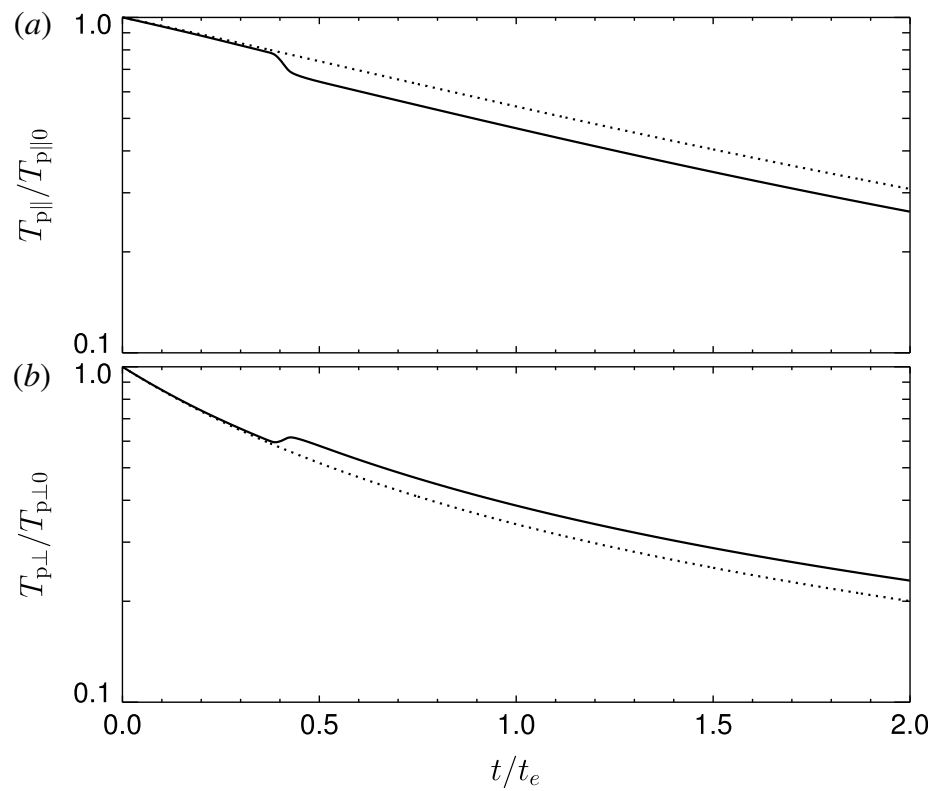

FIgURE 6. Evolution in 2D HEB simulation for $\theta_{B R}=32^{\circ}$ and $t_{e} \omega_{c p 0}=10^{4}$ : the proton parallel $T_{p \|}(a)$ and perpendicular $T_{p \perp}(b)$ as functions of time. The dotted curves show the corresponding double-adiabatic predictions.

time. Figure $7(b, c)$ show colour scale plots of the fluctuating magnetic energy $\delta B^{2}$ as a function of time and the wave vector $\boldsymbol{k} d_{p}$ and as a function of time and angle $\theta_{k B}$ between the ambient magnetic field and the wave vector, respectively. As the 


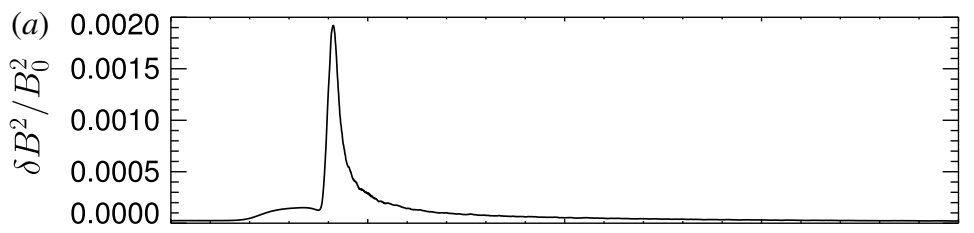

(b)

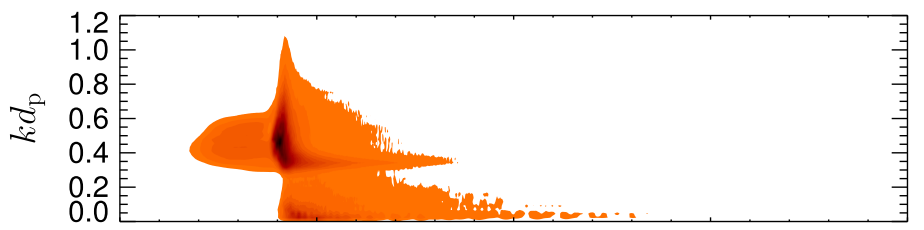

(c)

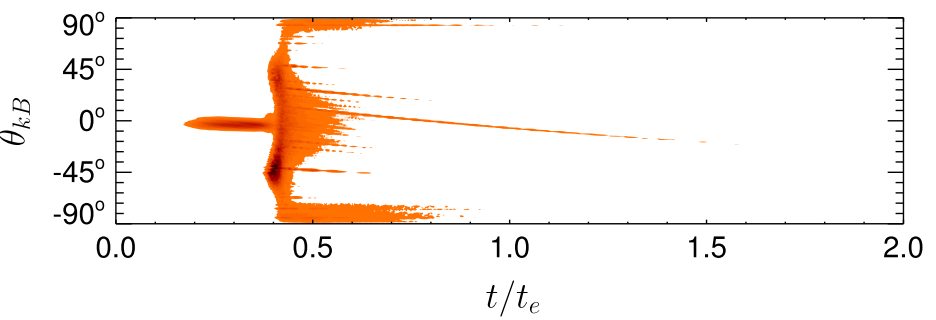

FIGURE 7. Evolution in 2D HEB simulation for $\theta_{B R}=32^{\circ}$ and $t_{e} \omega_{c p 0}=10^{4}$. (a) Fluctuating magnetic energy $\delta B^{2} / B_{0}^{2}$ as a function of time. $(b)$ Colour scale plot of the fluctuating magnetic energy $\delta B^{2}$ as a function of time and the wave vector $\boldsymbol{k}$. (c) Colour scale plot of the fluctuating magnetic energy $\delta B^{2}$ as a function of time and angle $\theta_{k B}$.

system expands, the plasma becomes unstable with respect to the parallel fire hose and relatively weak $\left(\delta B / B_{0} \sim 0.01\right)$ quasi-parallel $\left(\theta_{k B} \sim 0^{\circ}\right)$ propagating modes appear at proton scales $\left(k \sim 0.5 d_{p}^{-1}\right)$ for $0.2 \lesssim t / t_{e} \lesssim 0.4$. Around $t \sim 0.4$, oblique fire hose wave activity appears and mostly disappears quite rapidly (on a time scale $\sim 0.1 t_{e}$ ), similarly to the case with the radial magnetic field.

Figure 5 reveals a discrepancy between the linear prediction based on the bi-Maxwellian approximation and the simulation results. As in the case of slower expansion ( $\$ 2.3)$, the linear theory based on the bi-Maxwellian proton velocity distribution function predicts that the system is all the time stable with respect to the oblique fire hose, whereas the simulation results clearly exhibit a wave activity driven by this instability. This is connected with the resonant character of the instability (Gary 1993); its growth rate depends on the details (gradients) of the particle velocity distribution function. Figure 8 shows the maximum growth rate $\gamma_{\max }$ as a function of time for the parallel $(a)$ and oblique $(b)$ fire hose instabilities. The solid lines display the linear prediction calculated from the distribution function observed in the code (cf. Hellinger \& Trávníček 2011), whereas the dashed line shows the results for bi-Maxwellian velocity distribution functions that have the observed parallel and perpendicular proton temperatures.

Figure 8 clearly demonstrates that the growth rates calculated from the velocity distribution functions can widely differ from those calculated from bi-Maxwellian distribution functions having the same temperatures (cf. Isenberg 2012). For the parallel fire hose the two growth rates are the same only initially, $t \lesssim 0.15 t_{e}$. As the parallel fire hose activity increases, protons are scattered mainly in the resonant region of the velocity distribution function, giving rise to a quasi-linear plateau-like distribution. This plateau does not strongly influence the macroscopic moments, so 

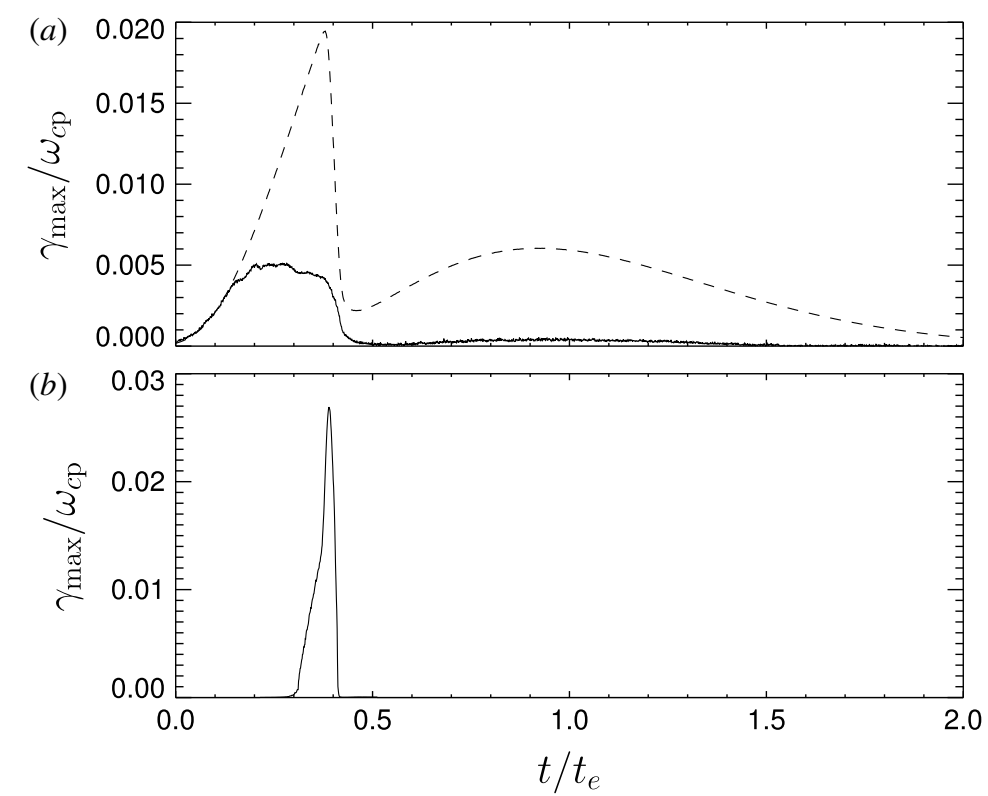

FIgURE 8. Evolution in 2D HEB simulation for $\theta_{B R}=32^{\circ}$ and $t_{e} \omega_{c p 0}=10^{4}$ : the maximum growth rate $\gamma_{\max }$ as a function of time for the parallel $(a)$ and oblique (b) fire hose instabilities. The solid lines display the linear prediction calculated from the proton velocity distribution function, whereas the dashed line shows the results for a bi-Maxwellian velocity distribution function corresponding to the parallel and perpendicular pressures.

that the linear prediction based on the bi-Maxwellian shape gives much stronger growth rates. On the other hand, the linear theory based on the instantaneous velocity distribution predicts an important growth rate for the oblique fire hose instability for $0.3 t_{e} \lesssim t \lesssim 0.4 t_{e}$, whereas in the corresponding bi-Maxwellian case this mode is stable. This behaviour is caused by the interplay between the expansion and the fire hose instabilities. The expansion affects the whole distribution function, whereas the two fire hose instabilities are resonant and interact preferably with the particles from their resonant regions which are different; the parallel fire hose interacts through the anomalous cyclotron resonance, whereas the oblique fire hose interacts through the cyclotron resonance (and the Landau resonance after the branch change, cf. Hellinger \& Trávníček 2006). As the parallel fire hose is stabilized through the modification of the distribution function in the vicinity of its resonant region, the rest of the distribution function is almost unaffected and may destabilize the oblique fire hose. At the same time, the parallel fire hose reduces the anisotropy on the macroscopic level so that in the corresponding bi-Maxwellian plasma the oblique fire hose is stabilized. The simulation results are in agreement with the linear prediction based on the instantaneous velocity distribution functions; the parallel fire hose activity is saturated around $t \sim 0.3 t_{e}$, the oblique fire hose wave activity has a maximum amplitude around $t \sim 0.4 t_{e}$, and after $t \sim 0.4 t_{e}$ all the wave activity decays.

The calculation of the linear growth rate is based on discrete velocity distribution functions calculated in the code over the whole simulation box. The distribution function is evaluated on a $512 \times 512$ grid in the velocity space $\left(v_{\|}, v_{\perp}\right)$ with respect to the ambient magnetic field. The maximum parallel and perpendicular velocities 

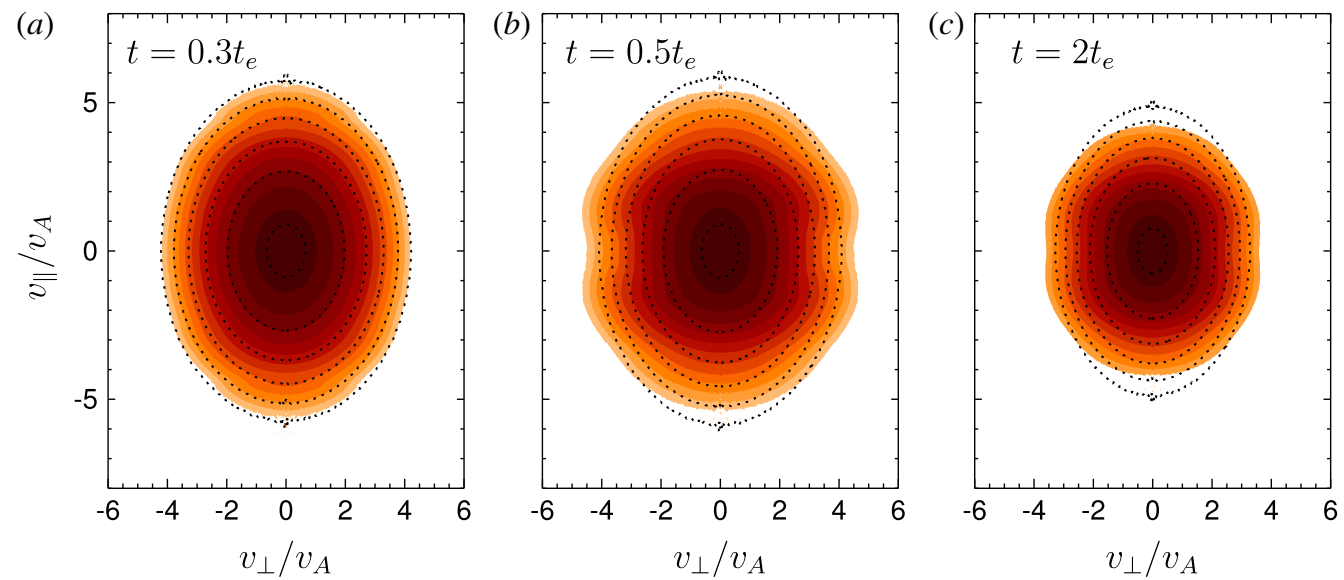

FIGURE 9. Evolution in 2D HEB simulation for $\theta_{B R}=32^{\circ}$ and $t_{e} \omega_{c p 0}=10^{4}$ : colour scale plots of the proton velocity distribution functions as functions of $v_{\perp}$ and $v_{\|}$(normalized to $v_{A}$ ) at $(a) t=0.3 t_{e}$, (b) $t=0.5 t_{e}$, and (c) $t=2 t_{e}$. The dotted contours display the doubleadiabatic prediction.

(and consequently the velocity grid size) are determined in the code by the maximum proton velocities. The finite number of particles per cell and the discretization are sources of different errors. In the present case, we estimate the error of the growth rate determination from the velocity distribution function to be of the order $10^{-4} \omega_{c p}$, so that the values in figure 8 are physically relevant.

The actual shape of the proton velocity distribution function is shown in figure 9, which displays colour scale plots of the distribution functions as functions of $v_{\perp}$ and $v_{\|}$(normalized to the Alfvén velocity $v_{A}$ ) at $(a) t=0.3 t_{e},(b) t=0.5 t_{e}$, and $(c)$ $t=2 t_{e}$. The dotted contours display the double-adiabatic prediction for comparison (this plot was obtained from the velocity distribution function at $t=0$ and rescaled using Equation $(2.1 a, b)$ ). At $t=0.3 t_{e}$, there are small (hardly discernible) differences between the distribution function and the CGL prediction around $\left|v_{\|}\right| \sim 0.4 v_{A}$. At $t=0.5 t_{e}$, when most of the wave activity driven by the two fire hoses is damped, there are clear differences between the distribution function and the CGL prediction. Afterwards, the distribution function essentially only rescales, following the CGL prediction.

\section{Discussion}

We presented three two-dimensional hybrid expanding box simulations of the competition between the expansion-driven parallel proton temperature anisotropy and fire hose instabilities. We used similar plasma parameters and varied the expansion time scale and the (initial) angle between the ambient magnetic field and the radial direction. The simulation results of the three simulations have similar properties; as the expansion drives $T_{p \|}>T_{p \perp}$, the system becomes unstable with respect the dominant parallel fire hose instability. This instability is generally unable to counteract the induced anisotropization and the system becomes eventually unstable with respect to the oblique fire hose. This instability efficiently reduces the anisotropy and the system becomes stable, while a significant part of the generated electromagnetic fluctuations is damped to protons. As long as the magnetic field is sufficiently close 
to the radial direction, this evolution repeats itself and the electromagnetic fluctuations accumulate. For a sufficiently oblique magnetic field the expansion drives $T_{p \perp}>T_{p \|}$ and brings the system to the stable region with respect to the fire hose instabilities, where the generated wave activity is damped. This evolution may eventually lead to instabilities driven by $T_{p \perp}>T_{p \|}$ (Trávníček et al. 2007). For slower driver (longer expansion time) and/or oblique magnetic field, the system enters less far into the unstable region and generates weaker wave amplitudes. Starting with a sufficiently oblique magnetic field, the system may completely avoid the fire hose instabilities. The simulation results also indicate that the assumption of bi-Maxwellian velocity distribution functions may lead to wrong results, as both the fire hoses are resonant and the simulation results show that the linear growth rate is sensitive to the details of the proton distribution function (cf. Hellinger \& Trávníček 2011). We expect similar results for alpha particle driven fire hose instabilities (Maruca et al. 2012; Verscharen, Bourouaine \& Chandran 2013; Matteini et al. 2015). A differential velocity between different ion species is another source of free energy for instabilities (Matteini et al. 2013). For electromagnetic instabilities the important parameter is the ratio between the differential and Alfvén velocities (Gary 1993; Daughton \& Gary 1998). Similar to the case of the parallel temperature anisotropy, the double-adiabatic approximation predicts that this ratio increases with the radial distance for the magnetic field that is not too oblique with respect to the magnetic field (Matteini et al. 2012). For a sufficiently oblique magnetic field this ratio decreases owing to the rotational force (Verscharen et al. 2015).

The present simulation results are obtained in a collisionless, homogeneous plasma where Coulomb collisions and the presence of an important turbulent solar wind activity (Bruno \& Carbone 2013) are neglected. We note that a behaviour similar to that presented here was also seen in weakly collisional plasmas (Hellinger \& Trávníček 2015) and simulation results of Hellinger et al. (2015) indicate that kinetic instabilities, such as the oblique fire hose, co-exist with fully developed turbulence. Moreover, similar results were also obtained for a completely different driver (velocity shear) of the temperature anisotropy (Kunz, Schekochihin \& Stone 2014). Consequently, our results are quite robust and applicable to a wide range of space or astrophysical plasmas.

In the context of the present work, it is interesting to briefly discuss the results of Matteini et al. (2007). The observed change of proton behaviour around 1 a.u. in the $\left(\beta_{p \|}, T_{p \perp} / T_{p \|}\right)$ plane is likely partly connected with the change of the expansion-driven anisotropization around $\theta_{B R} \sim 45^{\circ}$ that corresponds roughly to 1 a.u. However, these observations also indicate that $\beta_{p \|}$ increases with the radial distance even beyond 1 a.u., whereas the double-adiabatic approximation predicts that it should decrease then. This is probably connected with the fact that the solar wind protons do not generally follow the double-adiabatic prediction since they are continuously heated, likely owing to the turbulent cascade. More work is needed to understand the behaviour of turbulence in the expanding solar wind with an oblique magnetic field, the resulting proton energization, and the role of the fire hose instabilities in such a complex system.

\section{Acknowledgements}

The author acknowledges grant 15-10057S of the Grant Agency of the Czech Republic and projects RVO:67985815 and RVO:68378289. 


\section{REFERENCES}

Bale, S. D., Kasper, J. C., Howes, G. G., Quataert, E., Salem, C. \& Sundkvist, D. 2009 Magnetic fluctuation power near proton temperature anisotropy instability thresholds in the solar wind. Phys. Rev. Lett. 103, 211101.

Bourouaine, S., Verscharen, D., Chandran, B. D. G., Maruca, B. A. \& Kasper, J. C. 2013 Limits on alpha particle temperature anisotropy and differential flow from kinetic instabilities: solar wind observations. Astrophys. J. Lett. 777, L3.

Bruno, R. \& CARbone, V. 2013 The solar wind as a turbulence laboratory. Living Rev. Solar Phys. $10,2$.

Chew, G. F., Goldberger, M. L. \& Low, F. E. 1956 The Boltzmann equation and the one fluid hydromagnetic equations in the absence of particle collisions. Proc. R. Soc. Lond. A 236, $112-118$.

Cranmer, S. R., Matthaeus, W. H., Breech, B. A. \& Kasper, J. C. 2009 Empirical constraints on proton and electron heating in the fast solar wind. Astrophys. J. 702, 1604-1614.

DAughton, W. \& GARY, S. P. 1998 Electromagnetic proton/proton instabilities in the solar wind. J. Geophys. Res. 103, 20613-20620.

GARY, S. P. 1993 Theory of Space Plasma Microinstabilities. Cambridge University Press.

Gary, S. P., Li, H., O'Rourke, S. \& Winske, D. 1998 Proton resonant firehose instability: temperature anisotropy and fluctuating field constraints. J. Geophys. Res. 103, 14567-14574.

Gary, S. P., Neagu, E., Skoug, R. M. \& Goldstein, B. E. 1999 Solar wind electrons: parametric constraints. J. Geophys. Res. 104, 19843-19850.

Gary, S. P., Skoug, R. M., Steinberg, J. T. \& Smith, C. W. 2001 Proton temperature anisotropy constraint in the solar wind: ACE observations. Geophys. Res. Lett. 28, 2759-2763.

Grappin, R. \& Velli, M. 1996 Waves and streams in the expanding solar wind. J. Geophys. Res. 101, 425-444.

Grappin, R., Velli, M. \& Mangeney, A. 1993 Nonlinear-wave evolution in the expanding solar wind. Phys. Rev. Lett. 70, 2190-2193.

Hellinger, P. \& Matsumoto, H. 2000 New kinetic instability: oblique Alfvén fire hose. J. Geophys. Res. 105, 10519-10526.

Hellinger, P. \& Matsumoto, H. 2001 Nonlinear competition between the whistler and Alfvén fire hoses. J. Geophys. Res. 106, 13215-13218.

Hellinger, P., Matteini, L., Landi, S., Verdini, A., Franci, L. \& Trávníček, P. M. 2015 Kinetic plasma turbulence and instabilities in the expanding solar wind. Astrophys. J. Lett. 811, L32.

Hellinger, P. \& TRÁVníč́EK, P. 2005 Magnetosheath compression: role of characteristic compression time, alpha particle abundances and alpha/proton relative velocity. J. Geophys. Res. 110, A04210.

Hellinger, P. \& Trávníček, P. 2006 Parallel and oblique proton fire hose instabilities in the presence of alpha/proton drift: hybrid simulations. J. Geophys. Res. 111, A01107.

Hellinger, P. \& TRÁvníček, P. M. 2008 Oblique proton fire hose instability in the expanding solar wind: hybrid simulations. J. Geophys. Res. 113, A10109.

Hellinger, P. \& TrávníčeK, P. M. 2011 Proton core-beam system in the expanding solar wind: hybrid simulations. J. Geophys. Res. 116, A11101.

Hellinger, P. \& Trávníčé, P. M. 2014 Solar wind protons at 1 AU: trends and bounds, constraints and correlations. Astrophys. J. Lett. 784, L15.

Hellinger, P. \& TRÁVníČEK, P. M. 2015 Proton temperature-anisotropy-driven instabilities in weakly collisional plasmas: hybrid simulations. J. Plasma Phys. 81, 305810103.

Hellinger, P., Trávníček, P., Kasper, J. C. \& Lazarus, A. J. 2006 Solar wind proton temperature anisotropy: linear theory and WIND/SWE observations. Geophys. Res. Lett. 33, L09101.

Hellinger, P., Trávníček, P. M., Štverák, Š., Matteini, L. \& Velli, M. 2013 Proton thermal energetics in the solar wind: Helios reloaded. J. Geophys. Res. 118, 1351-1365. 
Hellinger, P., Velli, M., Trávníček, P., Gary, S. P., Goldstein, B. E. \& Liewer, P. C. 2005 Alfvén wave heating of heavy ions in the expanding solar wind: hybrid simulations. J. Geophys. Res. 110, A12109.

Hollweg, J. V. \& Isenberg, P. A. 2002 Generation of the fast solar wind: a review with emphasis on the resonant cyclotron interaction. J. Geophys. Res. 107, 1147.

IsENBERG, P. A. 2012 A self-consistent marginally stable state for parallel ion cyclotron waves. Phys. Plasmas 19, 032116.

Kasper, J. C., Lazarus, A. J., Gary, S. P. \& Szabo, A. 2003 Solar wind temperature anisotropies. In Solar wind ten (ed. M. Velli, R. Bruno \& F. Malara), vol. 679, pp. 538-541. AIP.

Kunz, M. W., Schekochinin, A. A. \& Stone, J. M. 2014 Firehose and mirror instabilities in a collisionless shearing plasma. Phys. Rev. Lett. 112, 205003.

Lacombe, C., Alexandrova, O., Matteini, L., Santolík, O., Cornilleau-Wehrlin, N., Mangeney, A., De Conchy, Y. \& Maksimovic, M. 2014 Whistler mode waves and the electron heat flux in the solar wind: cluster observations. Astrophys. J. 795, 5.

MARSCH, E. 2006 Kinetic physics of the solar corona and solar wind. Living Rev. Solar Phys. 3, http://www.livingreviews.org/lrsp-2006-1.

MARSCH, E., Ao, X.-Z.\& TU, C.-Y. 2004 On the temperature anisotropy of the core part of the proton velocity distribution function in the solar wind. J. Geophys. Res. 109, A04102.

MarucA, B. A., KASPer, J. C. \& GARY, S. P. 2012 Instability-driven limits on helium temperature anisotropy in the solar wind: observations and linear Vlasov analysis. Astrophys. J. 748, 137.

Matteini, L., Hellinger, P., Goldstein, B. E., Landi, S., Velli, M. \& Neugebauer, M. 2013 Signatures of kinetic instabilities in the solar wind. J. Geophys. Res. 118, 2771-2782.

Matteini, L., Hellinger, P., Landi, S., TrávníčeK, P. M. \& Velli, M. 2012 Ion kinetics in the solar wind: coupling global expansion to local microphysics. Space Sci. Rev. 172, 373-396.

Matteini, L., Hellinger, P., Schwartz, S. J. \& Landi, S. 2015 Firehose instability driven by alpha particle temperature anisotropy. Astrophys. J. 812, 13.

Matteini, L., Landi, S., Hellinger, P., Pantellini, F., Maksimovic, M., Velli, M., Goldstein, B. E. \& MARSCH, E. 2007 The evolution of the solar wind proton temperature anisotropy from 0.3 to 2 a.u. Geophys. Res. Lett. 34, L20105.

Matteini, L., Landi, S., Hellinger, P. \& Velli, M. 2006 Parallel proton fire hose instability in the expanding solar wind: hybrid simulations. J. Geophys. Res. 111, A10101.

Matthews, A. 1994 Current advance method and cyclic leapfrog for 2D multispecies hybrid plasma simulations. J. Comput. Phys. 112, 102-116.

PARKER, E. N. 1958 Dynamics of the interplanetary gas and magnetic fields. Astrophys. J. 128, 664-676.

Servidio, S., Valentini, F., Perrone, D., Greco, A., Califano, F., Matthaeus, W. H. \& Veltri, P. 2015 A kinetic model of plasma turbulence. J. Plasma Phys. 81, 325810107.

Štverák, Š., Trávníček, P., Maksimovic, M., Marsch, E., Fazakerley, A. \& Scime, E. E. 2008 Electron temperature anisotropy constraints in the solar wind. J. Geophys. Res. 113, A03103.

Trávníček, P., Hellinger, P., Taylor, M. G. G. T., Escoubet, C. P., Dandouras, I. \& LUCEK, E. 2007 Magnetosheath plasma expansion: hybrid simulations. Geophys. Res. Lett. 34, L15104.

Verscharen, D., Bourouaine, S. \& Chandran, B. D. G. 2013 Instabilities driven by the drift and temperature anisotropy of alpha particles in the solar wind. Astrophys. J. 773, 163.

Verscharen, D., Chandran, B. D. G., Bourouaine, S. \& Hollweg, J. V. 2015 Deceleration of alpha particles in the solar wind by instabilities and the rotational force: implications for heating, azimuthal flow, and the Parker spiral magnetic field. Astrophys. J. 806, 157.

Wicks, R. T., Matteini, L., Horbury, T. S., Hellinger, P. \& Roberts, A. D. 2013 Temperature anisotropy instabilities; combining plasma and magnetic field data at different distances from the sun. Proc. 13th Intl Solar Wind Conf. vol. 1539. pp. 303-306. AIP. 